\title{
Balancing Self-Healing and Shape Stability in Dynamic Covalent Photoresins for Stereolithography 3D Printing
}

\author{
Alejandra Durand-Silva, Karen P. Cortés-Guzmán, Rebecca M. Johnson, Sachini D. Perera, \\ Ronald A. Smaldone*
}

Department of Chemistry and Biochemistry, University of Texas at Dallas, 800 West Campbell Road, Richardson, Texas 75080, United States

*email: ronald.smaldone@utdallas.edu

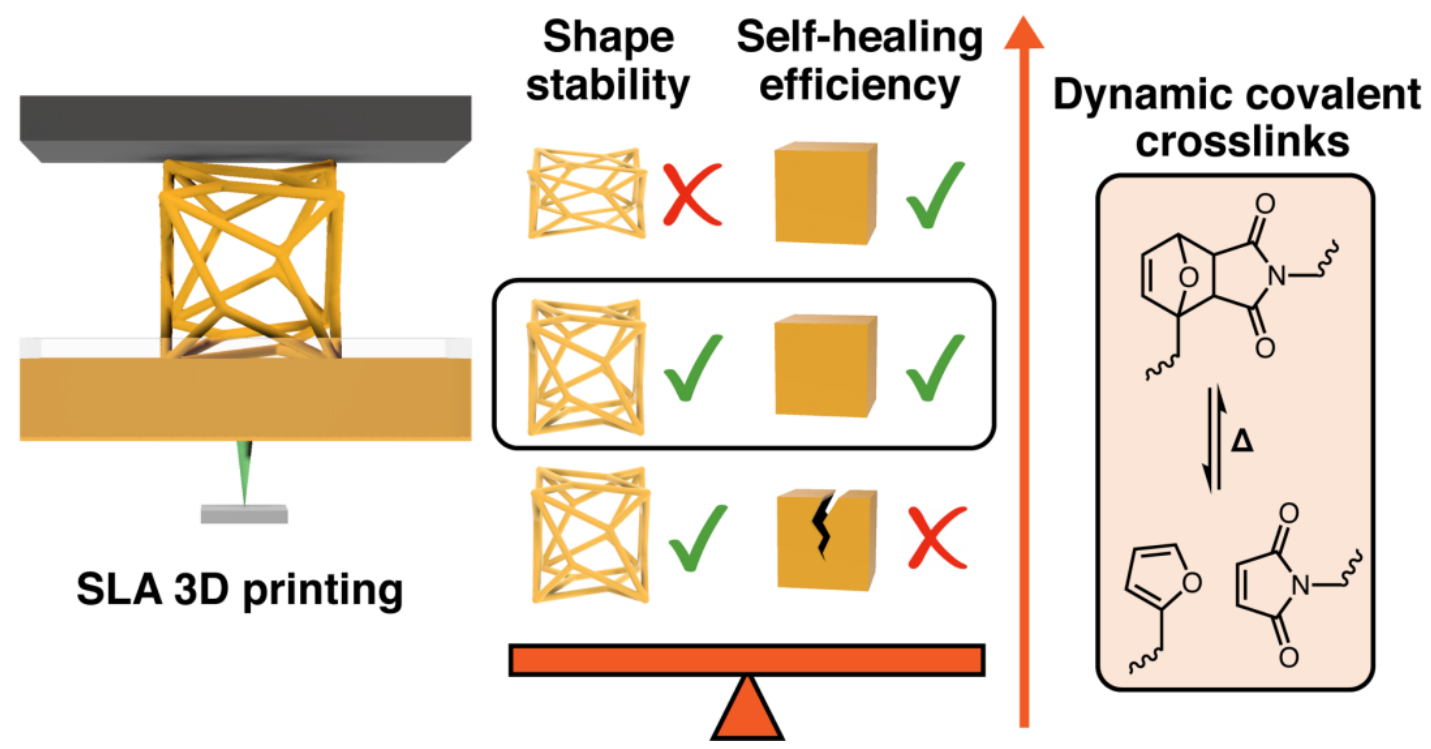

\begin{abstract}
Dynamic covalent bonds impart new properties to 3D printable materials that help to establish 3D printing as an accessible and efficient manufacturing technique. Here, we studied the effect of a thermally reversible Diels-Alder crosslinker on the shape stability of photoprintable resins and their self-healing properties. Resins containing different concentrations of dynamic covalent crosslinks in a polyacrylate network showed that the content of dynamic crosslinks plays a key role in balancing shape stability with self-healing ability. The shape stability of the printed objects was evaluated by measuring the dimensional changes after thermal treatment. The selfhealing efficiency of the 3D printed resins was characterized with a scratch test and tensile testing. A dynamic covalent crosslink concentration of $1.8 \mathrm{~mol} \%$ was enough to provide $99 \%$ self-healing efficiency without disrupting the shape stability of the printed objects. Our work shows the potential of dynamic covalent bonds in broadening the availability of 3D printable materials that are compatible with vat photopolymerization.
\end{abstract}


INTRODUCTION. Three-dimensional printing (3DP) is an advanced processing technique that builds objects from digital models by adding polymers layer-by-layer. 3DP has many advantages over other manufacturing techniques, such as the ability to produce custom designs without the need for additional tools (e.g., molds), and significantly reduced manufacturing waste compared with subtractive methods like milling. ${ }^{1,2}$ Several 3DP methods, such as extrusion and vat photopolymerization, have been developed to process thermoplastics, thermosets or soft materials. ${ }^{3,4}$ However, it remains a challenge to incorporate advanced functionality, such as selfhealing, into 3D printable polymers without affecting their processability, shape stability, or printability.

One key issue with 3DP is that the energy cost of printed objects is higher than those made with conventional manufacturing. ${ }^{5-7}$ In some cases, the selection of materials for 3DP may reduce the energy consumption, ${ }^{8,9}$ however, this approach may not be suitable for all printing methods. Hence, other sustainability aspects, such as increasing the lifespan of printed materials, are important to offset the energy cost. One way to achieve this is to incorporate self-healing capability into printable materials, which can extend their lifecycle in comparison with conventional plastics. Self-healing polymers are a class of materials that recover their mechanical strength after being cracked or damaged ${ }^{10}$ by using either extrinsic compartmentalized healing agents, ${ }^{11}$ or dynamic covalent or non-covalent interactions that are intrinsic to the polymer network. ${ }^{12,13}$ Imparting 3D printable polymers with self-healing properties increases their reliability as structural materials by enabling repair when cracks appear, preventing major failure and increasing the lifetime of the polymer. For this reason, developing printable self-healing polymers is a promising alternative to address our current overdependence on plastics and limited recycling capability, ${ }^{14-16}$ but also to make 3DP more sustainable.

The thermally reversible furan-maleimide Diels-Alder (fmDA) reaction has been extensively used for this purpose. ${ }^{17-22}$ Previously, our group has developed fmDA-based 3D printable materials for extrusion techniques with improved isotropy, taking advantage of the ability of the fmDA adduct to revert and reform throughout the printing process. ${ }^{23-25}$ However, since these materials consisted of mostly thermoplastic blends, they will lose their shape if heated for self-healing. ${ }^{23,24}$ Even extrusionprinted thermoset polymers containing fmDA adducts cannot efficiently retain their shape due to their intrinsic ability to melt at retro Diels-Alder temperatures, leading to deformations and rough surface finish. ${ }^{25}$ Therefore, a balance in between shape stability, thermal stability and dynamic covalent bonds is needed to print reliable self-healing materials based on fmDA adducts. Compared to extrusion methods, vat photopolymerization 3DP techniques, such as stereolithography (SLA), and digital light projection (DLP), are capable of higher resolution prints $(25 \mu \mathrm{m})$ and can create objects from thermoset polymers with improved quality and thermal stability, due to the formation of covalent bonds in between layers. This process is typically based on the free-radical polymerization of acrylate-based monomers and oligomers, that are formulated in a resin with photoinitiators. ${ }^{26,27}$ Although several extrudable materials have been developed to take advantage of dynamic covalent bonds, so far, examples with photoprinting remain limited. ${ }^{28,29}$

To study the effect of dynamic covalent crosslinks in the shape stability and self-healing ability of 3D printable thermosets, we prepared photoreactive resins with an acrylated fmDA crosslinker and printed them using SLA (Figure 1). By varying the concentration of fmDA crosslinks, we aimed to provide enough dynamic crosslink density to allow for self-healing under thermal treatment, without affecting the stability of the printed shapes. We evaluated the shape stability of these 3D printed networks throughout thermal treatment, as well as their self-healing efficiency with scratch and tensile tests. Our findings show that optimizing the content of dynamic crosslinks is necessary to achieve efficient self-healing (99\%) without affecting the network integrity and quality of the printed shape. 


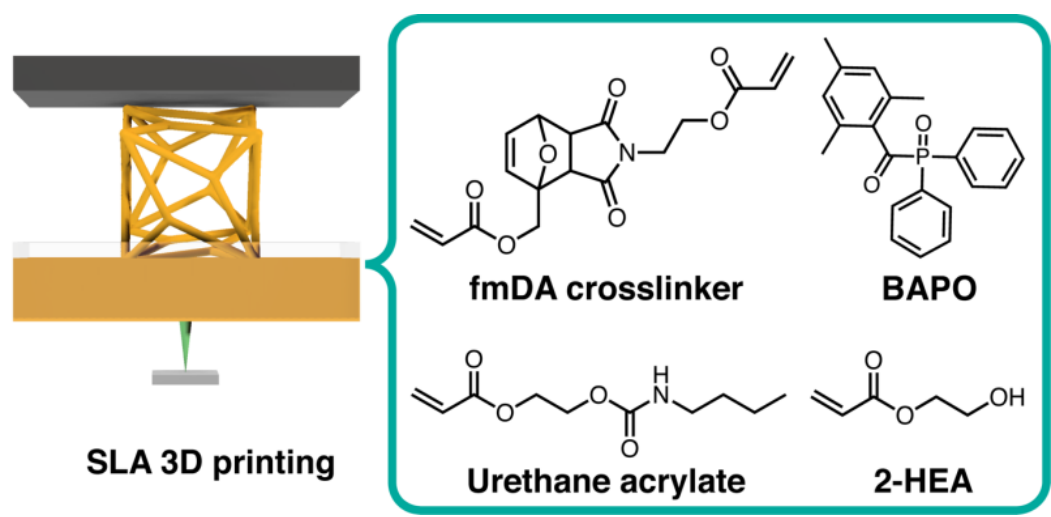

Figure 1. Formulation of self-healing SLA resins. Dynamic covalent crosslinking is provided by a fmDA diacrylated crosslinker; a urethane acrylate (Genomer 1122) and 2-hydroxyethyl acrylate (2HEA) are used as reactive diluents, and phenylbis(2,4,6-trimethylbenzoyl)phosphine oxide (BAPO) as the photoinitiator.

RESULTS AND DISCUSSION. The dynamic covalent resins were prepared using different weight percent concentrations of a fmDA acrylated crosslinker, 30 a urethane acrylate (Genomer $(1122$ ) and 2-hydroxyethyl acrylate (2-HEA) as diluents, and phenylbis(2,4,6-trimethylbenzoyl)phosphine oxide (BAP0) as a photoinitiator (Figure 1, Table S1). All resins were printed in a Formlabs® 2 SLA printer at a resolution of $100 \mu \mathrm{m}$, without supports. The resins were easily 3D printed after formulation without further optimization due to the high concentration of 2-HEA, a low viscosity photoreactive diluent. The curing efficiency of the fmDA resins was assessed by Fourier-transform infrared spectroscopy (FTIR) of their corresponding 3D printed films, after post curing steps. The signals corresponding to the vinyl $\mathrm{C}$-H stretch in the acrylated monomers disappeared after post curing, indicating complete conversion (Figure S6).

The fmDA adduct is reported to be reversible at temperatures around $110-130{ }^{\circ} \mathrm{C} .17,19$ To determine a suitable range of self-healing temperatures, the thermal stability of the polymer networks was evaluated with thermogravimetric analysis (TGA), which shows degradation of the polymer networks above $150{ }^{\circ} \mathrm{C}$ (Figure S7). Therefore, the self-healing tests were performed at $110{ }^{\circ} \mathrm{C}$ to avoid the degradation of the material. Differential scanning calorimetry (DSC) was used to characterize the glass transition temperature ( $\mathrm{T}_{\mathrm{g}}$, Figure $\mathrm{S} 8$ ). The $\mathrm{T}_{\mathrm{g}}$ of the fmDA resins was observed around room temperature, increasing proportionally with the fmDA crosslinker concentration, varying from $16{ }^{\circ} \mathrm{C}$ for the $2.5 \% \mathrm{fmDA}$ resin, up to $30{ }^{\circ} \mathrm{C}$ for the $20 \%$ fmDA resin (Table S2).

To test the shape stability of fmDA networks under thermal treatment for self-healing conditions, 1 x $2 \times 6 \mathrm{~mm}$ rectangular prisms were 3D printed with each formulation and clamped to apply the required pressure in a self-healing test ${ }^{31}$ throughout the $2 \times 6 \mathrm{~mm}$ area, perpendicular to the thickness dimension. Then, they were subjected to thermal treatment at $110{ }^{\circ} \mathrm{C}$ for $24 \mathrm{~h}$, followed by $80{ }^{\circ} \mathrm{C}$ for $24 \mathrm{~h}$. All the formulations showed a decrease in thickness, with a maximum decrease of $19 \%$ for the $20 \%$ fmDA resin (Figure 2a, Table S3). In contrast to thickness, width and length increased in most samples, which is consistent with the pressure applied perpendicularly. The $20 \%$ fmDA resin also exhibited the largest deformation, increasing its width by $11.9 \%$, whereas the $15 \%$ fmDA resin showed the maximum increase in length of $6.7 \%$ (Figures $2 b-c$, Table S3). Overall, the dimensional variations of the 3D printed shapes were proportional to the concentration of the dynamic covalent crosslinker. To observe these variations in a larger scale, complex 3D printed shapes with 10, 15 and 20\% fmDA resins, which showed larger dimensional variations, were printed and subjected to a shape stability test, placing a glass slide on top of the samples to apply pressure during the thermal treatment. Only parts printed with the $20 \% \mathrm{fmDA}$ resin were unable to maintain their original shape (Figure $2 \mathrm{~d}$ ). 
The healing efficiency of the fmDA resins was qualitatively assessed with a scratch test. $10 \times 10 \mathrm{~mm}$ films of each formulation were printed and scratched with a razor blade, then subjected to thermal treatment, without any pressure. The healing efficiency was evaluated by heating the $\mathrm{m}$ at $110^{\circ} \mathrm{C}$ for $24 \mathrm{~h}$ followed by $80^{\circ} \mathrm{C}$ for $24 \mathrm{~h}$, to promote self-healing. Optical microscope pictures of the scratches were compared before and after thermal treatment (Figures 2e and S9). We observed larger deformations around the scratch in the softest materials, such as the 2.5 and $5 \%$ fmDA resins, likely due to lower crosslink density. The 2.5, 10 and 15\% fmDA resins demonstrated the lower scratch healing ability in comparison with the 5 and $20 \%$ fmDA resins. Under the microscope, the original surface of the films shows a pattern of the successive printed layers, which is expected due to the inherent anisotropy that results from the layer-by-layer process, ${ }^{32,33}$ but after the thermal treatment the surface quality appears to be improved into a smooth surface in all the samples.
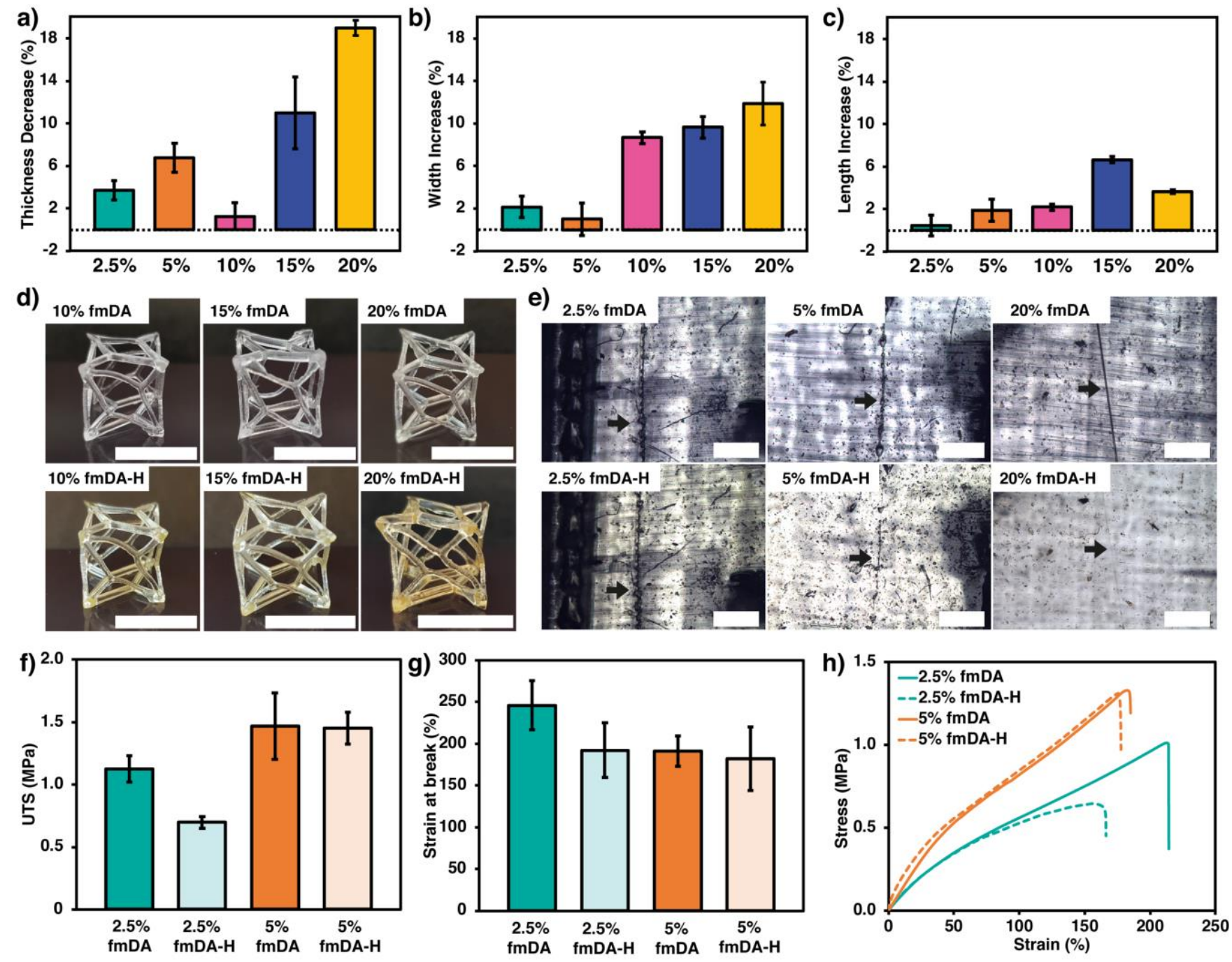

Figure 2. Shape stability tests after thermal treatment, with different weight percent concentrations of fmDA crosslinks a) Percentage thickness decrease, b) percentage width increase, c) percentage length increase. d) Complex 3D printed shapes with different concentrations of fmDA crosslinks before (top), and after thermal treatment (bottom), white scale bars represent $15 \mathrm{~mm}$. e) Optical microscope images of scratch self-healing test of 3D printed fmDA resins. Top images show the original scratched samples of 2.5, 5 and 20\% fmDA 3D printed resins. Bottom images show the same samples after healing (fmDA-H), white scale bars represent $500 \mu \mathrm{m}$. Tensile testing of 3D printed fmDA specimens: f) ultimate tensile strength (UTS), g) strain at break, and h) representative stress-strain curves of original (fmDA) and self-healed (fmDA-H) resins. 
To quantitively determine the healing efficiency of the fmDA resins, 3D printed ASTM D638 type 4 specimens of each formulation were cut in half, placed together in between a clamp and subjected to thermal treatment for self-healing. The mechanical properties of original and healed specimens were evaluated by tensile testing (Table 1). The best healing efficiency was observed in resins with the lowest concentration of fmDA crosslinks. The $2.5 \% \mathrm{fmDA}$ resin only recovered $62 \%$ of its original ultimate tensile strength (UTS), but the $5 \% \mathrm{fmDA}$ resin recovered up to $99 \%$ of its original UTS (Table 1, Figure 2f,). Following the same trend, the $2.5 \% \mathrm{fmDA}$ resin recovered only $78 \%$ of its original strain at break, whereas the $5 \% \mathrm{fmDA}$ resin recovered $95 \%$ of its original strain at break (Table 1, Figure $2 \mathrm{~g}$ ). Higher concentrations of fmDA crosslinks resulted in poor healing ability. The 10\% fmDA resin recovered only $10 \%$ of its original UTS and strain at break, and the $15 \%$ fmDA resin did not show any self-healing at all (Table 1, Figures S10-S11). The Young's modulus of all the samples before and after healing was proportional to the crosslinking density, increasing from $0.74 \mathrm{MPa}$ in the $2.5 \%$ fmDA resin to $8.37 \mathrm{MPa}$ in the $15 \% \mathrm{fmDA}$ resin, and it was not significantly diminished after self-healing treatment (Table 1, Figure S12).

Table 1. Mechanical properties of original and healed fmDA resins.

\begin{tabular}{|c|c|c|c|c|c|c|c|c|c|}
\hline Resin & UTS (MPa) & $\begin{array}{c}\text { UTS after } \\
\text { healing } \\
\text { (MPa) }\end{array}$ & $\begin{array}{c}\text { UTS } \\
\text { recovery } \\
(\%)\end{array}$ & $\begin{array}{c}\text { Strain at } \\
\text { break (\%) }\end{array}$ & $\begin{array}{c}\text { Strain at } \\
\text { break after } \\
\text { healing }(\%)\end{array}$ & $\begin{array}{l}\text { Strain at } \\
\text { break } \\
\text { recovery } \\
(\%)\end{array}$ & $\begin{array}{c}\text { Young's } \\
\text { modulus } \\
(\mathrm{MPa})\end{array}$ & $\begin{array}{c}\text { Young's } \\
\text { modulus after } \\
\text { healing } \\
\text { (MPa) }\end{array}$ & $\begin{array}{l}\text { Young's } \\
\text { modulus } \\
\text { recovery } \\
(\%)\end{array}$ \\
\hline $\begin{array}{l}2.5 \% \\
\text { fmDA }\end{array}$ & $1.12( \pm 0.10)$ & $0.70( \pm 0.05)$ & 62 & $246( \pm 29)$ & $192( \pm 32)$ & 78 & $0.74( \pm 0.04)$ & $0.72( \pm 0.06)$ & 97 \\
\hline $\begin{array}{l}5 \% \\
\text { fmDA }\end{array}$ & $1.47( \pm 0.27)$ & $1.45( \pm 0.13)$ & 99 & $191( \pm 18)$ & $182( \pm 38)$ & 95 & $1.19( \pm 0.07)$ & $1.73( \pm 0.72)$ & 146 \\
\hline $\begin{array}{l}10 \% \\
\text { fmDA }\end{array}$ & $4.94( \pm 0.70)$ & $0.49( \pm 0.16)$ & 10 & $128( \pm 18)$ & $13( \pm 2)$ & 10 & $3.58( \pm 0.56)$ & $4.19( \pm 1.30)$ & 117 \\
\hline $\begin{array}{l}15 \% \\
\text { fmDA }\end{array}$ & $4.99( \pm 0.76)$ & NO & NO & $10( \pm 7)$ & $\mathrm{NO}$ & NO & $8.37( \pm 0.43)$ & NO & NO \\
\hline
\end{tabular}

NO: not observed

Our observations show that the abundance of reversible bonds has an influence on the shape stability and the self-healing ability of the polymer network. Of all the evaluated formulations, we found that the 5\% fmDA resin has the best self-healing efficiency balanced with good shape stability. The poor self-healing ability of the $2.5 \%$ fmDA resin can be attributed to a low number of available dynamic crosslinks. Although the 10 and 15\% fmDA resins contain more dynamic crosslinks, they did not exhibit self-healing properties in the scratch and tensile tests (Figures S9-S11). Although the retro Diels-Alder reaction takes place above the $\mathrm{T}_{\mathrm{g}}$ of all fmDA resins (Table S2), the poor healing we observed suggests that an elevated $\mathrm{T}_{\mathrm{g}}$ may play a role in reducing the self-healing ability. ${ }^{12}$ However, the $20 \% \mathrm{fmDA}$ resin showed improved self-healing ability in the scratch test, compared to the 10 and $15 \%$ fmDA resins. Although it is detrimental for shape stability, the increased availability of dynamic covalent crosslinks in the $20 \%$ fmDA resin may improve the self-healing ability and overcome the negative effect of higher $\mathrm{T}_{\mathrm{g}}$ in the polymer chain mobility. 


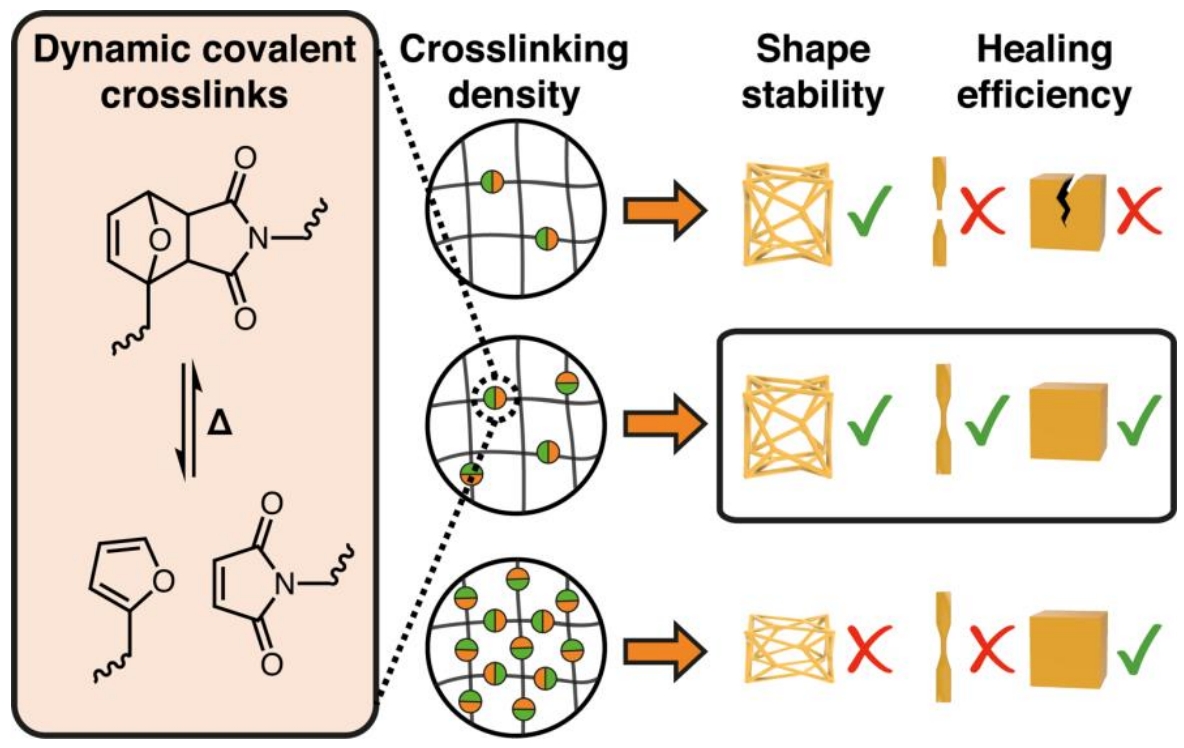

Figure 3. By varying the concentration of dynamic crosslinks in fmDA resins, it is possible to balance the self-healing efficiency and the shape stability of printed objects. These properties were evaluated by studying the amount of physical distortion in a printed object with fine features, and the recovery of mechanical strength after either fracture, or a surface scratch.

CONCLUSIONS. By evaluating SLA printable resins with different concentrations of dynamic covalent crosslinks, we demonstrate that the optimization of crosslinker content is important for balancing shape stability and healability of the printed polymer networks (Figure 3). In this work, only the $5 \%$ fmDA resin, which contains 1.8 mol \% of dynamic crosslinks, was successful in maintaining shape stability and self-healing efficiency. Our work shows that dynamic covalent photoresins can provide high resolution prints while maintaining excellent remendability properties and without damaging the shape stability upon thermal healing treatment. The incorporation of dynamic covalent chemistry in 3DP will help provide extended lifetimes to printed materials and empower the unique manufacturing capabilities of 3DP technology to be used more broadly, and most importantly, more sustainably.

ACKNOWLEDGMENTS. We acknowledge support from the Department of Energy's National Nuclear Security Agency, managed by Honeywell FM\&T (N000263171) and the Army Research Laboratory (W911NF-18-2-0035). A. D. S. and K. C. G. acknowledge the Consejo Nacional de Ciencia y Tecnología (CONACYT, Mexican Council of Science and Technology) for doctoral fellowships. We also acknowledge the Advanced Polymer Research Lab (APRL) at UT Dallas for access to facilities for the thermal characterization of polymers.

\section{REFERENCES}

1. Zastrow, M. 3D Printing Gets Bigger, Faster and Stronger. Nature 2020, 578, 20-23.

2. Lipson, H.; Kurman, M. Fabricated: The New World of 3D Printing; John Wiley \& Sons: Somerset, United States, 2013.

3. Gibson, I.; Rosen, D.; Stucker, B.; Khorasani, M. Additive Manufacturing Technologies, 3rd ed.; Springer: Cham, Switzerland 2021. 
4. $\quad$ Ligon, S. C.; Liska, R.; Stampfl, J.; Gurr, M.; Mülhaupt, R. Polymers for 3D Printing and Customized Additive Manufacturing. Chem. Rev. 2017, 117, 10212-10290.

5. Faludi, J.; Baumers, M.; Maskery, I.; Hague, R. Environmental Impacts of Selective Laser Melting: Do Printer, Powder, Or Power Dominate? J. Ind. Ecol. 2017, 21, S144-S156.

6. Faludi, J.; Bayley, C.; Bhogal, S.; Iribarne, M. Comparing Environmental Impacts of Additive Manufacturing vs. Traditional Machining Via Life-Cycle Assessment. Rapid Prototyp. J. 2015, 21, 14-33.

7. Huang, S. H.; Liu, P.; Mokasdar, A.; Hou, L. Additive Manufacturing and Its Societal Impact: A Literature Review. Int. J. Adv. Manuf. Technol. 2013, 67, 1191-1203.

8. Faludi, J.; Hu, Z.; Alrashed, S.; Braunholz, C.; Kaul, S.; Kassaye, L. Does Material Choice Drive Sustainability of 3D Printing? Int. J. Mech., Aero., Ind. Mechatr. Eng. 2015, 9, 216-223.

9. Faludi, J.; van Sice, C. M.; Shi, Y.; Bower, J.; Brooks, O. M. K. Novel Materials Can Radically Improve Whole-System Environmental Impacts of Additive Manufacturing. J. Cleaner Prod. 2019, 212, 1580-1590.

10. Wang, S.; Urban, M. W. Self-Healing Polymers. Nat. Rev. Mater. 2020, 5, 562-583.

11. White, S. R.; Sottos, N. R.; Geubelle, P. H.; Moore, J. S.; Kessler, M. R.; Sriram, S. R.; Brown, E. N.; Viswanathan, S. Autonomic Healing of Polymer Composites. Nature 2001, 409, 794-797.

12. Dahlke, J.; Zechel, S.; Hager, M. D.; Schubert, U. S. How to Design a Self-Healing Polymer: General Concepts of Dynamic Covalent Bonds and Their Application for Intrinsic Healable Materials. Adv. Mater. Interfaces 2018, 5, 1800051.

13. Herbst, F.; Döhler, D.; Michael, P.; Binder, W. H. Self-Healing Polymers via Supramolecular Forces. Macromol. Rapid Commun. 2013, 34, 203-220.

14. Borrelle, S. B.; Ringma, J.; Law, K. L.; Monnahan, C. C.; Lebreton, L.; McGivern, A.; Murphy, E.; Jambeck, J.; Leonard, G. H.; Hilleary, M. A.; Eriksen, M.; Possingham, H. P.; de Frond, H.; Gerber, L. R.; Polidoro, B.; Tahir, A.; Bernard, M.; Mallos, N.; Barnes, M.; Rochman, C. M. Predicted Growth in Plastic Waste Exceeds Efforts to Mitigate Plastic Pollution. Science 2020, $369,1515-1518$.

15. Schneiderman, D. K.; Hillmyer, M. A. 50th Anniversary Perspective: There Is a Great Future in Sustainable Polymers. Macromolecules 2017, 50, 3733-3749.

16. Fortman, D. J.; Brutman, J. P.; de Hoe, G. X.; Snyder, R. L.; Dichtel, W. R.; Hillmyer, M. A. Approaches to Sustainable and Continually Recyclable Cross-Linked Polymers. ACS Sustainable Chem. Eng. 2018, 6, 11145-11159.

17. Chen, X.; Dam, M. A.; Ono, K.; Mal, A.; Shen, H.; Nutt, S. R.; Sheran, K.; Wudl, F. A Thermally Re-Mendable Cross-Linked Polymeric Material. Science 2002, 295, 1698-1702.

18. Liu, Y. L.; Chuo, T. W. Self-Healing Polymers Based on Thermally Reversible DielsAlder Chemistry. Polym. Chem. 2013, 4, 2194-2205.

19. Foster, E. M.; Lensmeyer, E. E.; Zhang, B.; Chakma, P.; Flum, J. A.; Via, J. J.; Sparks, J. L.; Konkolewicz, D. Effect of Polymer Network Architecture, Enhancing Soft Materials Using Orthogonal Dynamic Bonds in an Interpenetrating Network. ACS Macro Lett. 2017, 6, 495499.

20. Schäfer, S.; Kickelbick, G. Double Reversible Networks: Improvement of Self-Healing in Hybrid Materials via Combination of Diels-Alder Cross-Linking and Hydrogen Bonds. Macromolecules 2018, 51, 6099-6110.

21. Karami, Z.; Zolghadr, M.; Zohuriaan-Mehr, M. J. Self-Healing Diels-Alder Engineered Thermosets. In Self-Healing Polymer-Based Systems; Elsevier, 2020; pp 209-233. 
22. Yuan, T.; Zhang, L.; Li, T.; Tu, R.; Sodano, H. A. 3D Printing of a Self-Healing, High Strength, and Reprocessable Thermoset. Polym. Chem. 2020, 11, 6441-6452.

23. Davidson, J. R.; Appuhamillage, G. A.; Thompson, C. M.; Voit, W.; Smaldone, R. A. Design Paradigm Utilizing Reversible Diels-Alder Reactions to Enhance the Mechanical Properties of 3D Printed Materials. ACS Appl. Mater. Interfaces 2016, 8, 16961-16966.

24. Appuhamillage, G. A.; Reagan, J. C.; Khorsandi, S.; Davidson, J. R.; Voit, W.; Smaldone, R. A. 3D Printed Remendable Polylactic Acid Blends with Uniform Mechanical Strength Enabled by a Dynamic Diels-Alder Reaction. Polym. Chem. 2017, 8, 2087-2092.

25. Yang, K.; Grant, J. C.; Lamey, P.; Joshi-Imre, A.; Lund, B. R.; Smaldone, R. A.; Voit, W. Diels-Alder Reversible Thermoset 3D Printing: Isotropic Thermoset Polymers via Fused Filament Fabrication. Adv. Funct. Mater. 2017, 27, 1700318.

26. Yee, D. W.; Greer, J. R. Three-dimensional Chemical Reactors: In Situ Materials Synthesis to Advance Vat Photopolymerization. Polym. Int. 2021, in press.

27. Bagheri, A.; Jin, J. Photopolymerization in 3D Printing. ACS Appl. Polym. Mater. 2019, $1,593-611$.

28. Li, X.; Yu, R.; He, Y.; Zhang, Y.; Yang, X.; Zhao, X.; Huang, W. Self-Healing Polyurethane Elastomers Based on a Disulfide Bond by Digital Light Processing 3D Printing. ACS Macro Lett. 2019, 8, 1511-1516.

29. Li, X.; Yu, R.; He, Y.; Zhang, Y.; Yang, X.; Zhao, X.; Huang, W. Four-Dimensional Printing of Shape Memory Polyurethanes with High Strength and Recyclability Based on Diels-Alder Chemistry. Polymer 2020, 200, 122532.

30. Heath, W. H.; Palmieri, F.; Adams, J. R.; Long, B. K.; Chute, J.; Holcombe, T. W.; Zieren, S.; Truitt, M. J.; White, J. L.; Willson, C. G. Degradable Cross-Linkers and Strippable Imaging Materials for Step-and-Flash Imprint Lithography. Macromolecules 2008, 41, 719-726.

31. De Alwis Watuthanthrige, N.; Ahammed, B.; Dolan, M. T.; Fang, Q.; Wu, J.; Sparks, J. L.; Zanjani, M. B.; Konkolewicz, D.; Ye, Z. Accelerating Dynamic Exchange and Self-Healing Using Mechanical Forces in Crosslinked Polymers. Mater. Horiz. 2020, 7, 1581-1587.

32. Gojzewski, H.; Guo, Z.; Grzelachowska, W.; Ridwan, M. G.; Hempenius, M. A.; Grijpma, D. W.; Vancso, G. J. Layer-by-Layer Printing of Photopolymers in 3D: How Weak Is the Interface? ACS Appl. Mater. Interfaces 2020, 12, 8908-8914.

33. Kim, H.; Han, S.; Seo, Y. Novel Dual-Curing Process for a Stereolithographically Printed Part Triggers a Remarkably Improved Interlayer Adhesion and Excellent Mechanical Properties. Langmuir 2020, 36, 9250-9258. 